Bull. Korean Math. Soc. 46 (2009), No. 5, pp. 949-966

DOI 10.4134/BKMS.2009.46.5.949

\title{
DIRAC EIGENVALUES ESTIMATES IN TERMS OF DIVERGENCEFREE SYMMETRIC TENSORS
}

\author{
Eui Chul Kim
}

Dedicated to Professor Thomas Friedrich on his 60th birthday

\begin{abstract}
We proved in [10] that Friedrich's estimate [5] for the first eigenvalue of the Dirac operator can be improved when a Codazzi tensor exists. In the paper we further prove that his estimate can be improved as well via a well-chosen divergencefree symmetric tensor. We study the geometric implication of the new first eigenvalue estimates over Sasakian spin manifolds and show that some particular types of spinors appear as the limiting case.
\end{abstract}

\section{Introduction}

Let $\left(M^{n}, g\right)$ be an $n$-dimensional closed Riemannian spin manifold. Let us denote by $\nabla$ the Levi-Civita connection on $\left(M^{n}, g\right)$ as well as the induced covariant derivative on the spinor bundle $\Sigma(M)$ and denote by $D$ the Dirac operator of $\left(M^{n}, g\right)$. Using a local orthonormal frame $\left(E_{1}, \ldots, E_{n}\right)$, we have the local formulas

$$
\nabla_{X} \psi=X(\psi)+\frac{1}{4} \sum_{i=1}^{n} E_{i} \cdot \nabla_{X} E_{i} \cdot \psi, \quad D \psi=\sum_{i=1}^{n} E_{i} \cdot \nabla_{E_{i}} \psi, \quad \psi \in \Gamma(\Sigma(M)),
$$

where the dot "." indicates the Clifford multiplication [6]. Associated to any nondegenerate symmetric $(0,2)$-tensor field $\beta$ on $\left(M^{n}, g\right)$, we define the $\beta$-twist $D_{\beta}$ of the Dirac operator $D$ by

$$
D_{\beta} \psi=\sum_{i=1}^{n} \beta^{-1}\left(E_{i}\right) \cdot \nabla_{E_{i}} \psi=\sum_{i=1}^{n} E_{i} \cdot \nabla_{\beta^{-1}\left(E_{i}\right)} \psi,
$$

where $\beta$ was identified with the induced $(1,1)$-tensor $\beta$ via $\beta(X, Y)=g(X, \beta(Y))$. In this paper, we are interested in two particular types of symmetric tensors, i.e., Codazzi tensors and divergencefree tensors: A symmetric (0,2)-tensor field $\beta$ is called

Received August 18, 2008.

2000 Mathematics Subject Classification. 53C25, 53C27.

Key words and phrases. Dirac operator, eigenvalues, divergencefree symmetric tensors. 
(i) a Codazzi tensor if $\left(\nabla_{X} \beta\right)(Y, Z)=\left(\nabla_{Y} \beta\right)(X, Z)$ holds for all vector fields $X, Y, Z$.

(ii) a divergencefree tensor if $\operatorname{div}(\beta)=\sum_{i=1}^{n}\left(\nabla_{E_{i}} \beta\right)\left(E_{i}\right)=0$.

Throughout the paper we fix some terminology.

Definition 1.1. Let $P$ be a first order self-adjoint elliptic operator on some closed Riemannian spin manifold. An eigenvalue $\lambda \in \mathbb{R}$ of $P$ is called the first eigenvalue if $\lambda^{2}$ is the smallest eigenvalue of $P^{2}$. An eigenspinor $\varphi$ of $P$ is called a first eigenspinor if its associated eigenvalue $\lambda$ is the first eigenvalue of $P$.

Well-known examples for the first eigenspinors of the Dirac operator are, e.g., parallel spinors [3, 12], Killing spinors [2, 3] and Kählerian Killing spinors [11].

We have recently proved in [10] the following two theorems.

Theorem A ([10]). Let $\left(M^{n}, g\right)$ be an $n$-dimensional closed Riemannian spin manifold and consider a nondegenerate Codazzi tensor $\beta$ such that $\operatorname{tr}\left(\beta^{-1}\right)=$ 0 vanishes identically. Denote by $\bar{g}$ the metric induced by $\beta$ via $\bar{g}(X, Y)=$ $g(\beta(X), \beta(Y))$ and by $\bar{D}$ the Dirac operator of $\bar{g}$. Let $\lambda_{1} \in \mathbb{R}$ and $\bar{\lambda}_{1} \in \mathbb{R}$ be the first eigenvalue of the Dirac operators $D$ and $\bar{D}$, respectively. Then we have

$$
\lambda_{1}^{2} \geq \inf _{M}\left\{\frac{n S}{4(n-1)}+\frac{n \bar{\lambda}_{1}^{2}}{(n-1)\left|\beta^{-1}\right|^{2}}+\frac{n \triangle F}{2(n-1) F}\right\},
$$

where $F: M^{n} \longrightarrow \mathbb{R}$ is a real-valued function defined by

$$
F=\left|\operatorname{det}\left(\beta^{-1}\right)\right| \cdot\left|\beta^{-1}\right|^{2},
$$

$\triangle F:=-(\operatorname{div} \circ \operatorname{grad})(F)$, and $S$ is the scalar curvature of $\left(M^{n}, g\right)$.

The limiting case of (1.1) occurs if and only if there exists a spinor field $\psi_{1}$ on $\left(M^{n}, g\right)$ with the following properties:

(i) The differential equation

$$
\nabla_{X} \psi_{1}=-\frac{\lambda}{n} X \cdot \psi_{1}-\frac{\bar{\lambda}}{\left|\beta^{-1}\right|^{2}} \beta^{-1}(X) \cdot \psi_{1}
$$

holds for some constants $\lambda, \bar{\lambda} \in \mathbb{R}$ and for all vector fields $X$.

(ii) $\psi_{1}$ is a first eigenspinor of both $D$ and $\bar{D}$.

Theorem B $([10])$. Let $\left(M^{n}, g\right)$ be an $n$-dimensional closed Riemannian spin manifold and consider a nondegenerate Codazzi tensor $\beta$. Let $\lambda_{1} \neq 0 \in \mathbb{R}$ and $\bar{\lambda}_{1} \in \mathbb{R}$ be the first eigenvalue of the Dirac operators $D$ and $\bar{D}$, respectively. Then, in the notations of Theorem A, we have

$$
\lambda_{1}^{2} \geq \inf _{M}\left\{\frac{S}{4(p+1)}-\frac{q \bar{\lambda}_{1}^{2}}{p+1}+\frac{\triangle F}{2(p+1) F}\right\},
$$

where the real-valued function $F: M^{n} \longrightarrow \mathbb{R}$ is defined by

$$
F=-\frac{\left|\operatorname{det}\left(\beta^{-1}\right)\right|}{q}
$$


and $p, q: M^{n} \longrightarrow \mathbb{R}$ are such bounded real-valued functions satisfying

$$
-\frac{1}{n}<p<0, \quad-\frac{1}{\left|\beta^{-1}\right|^{2}}<q<0,
$$

that solve the system of two linear equations

$$
n p+c\left(\operatorname{tr} \beta^{-1}\right) q=-1, \quad\left(\operatorname{tr} \beta^{-1}\right) p+c\left|\beta^{-1}\right|^{2} q=-c
$$

for some nonzero constant $c \neq 0 \in \mathbb{R}$.

The limiting case of (1.3) occurs if and only if there exists a spinor field $\psi_{1}$ on $\left(M^{n}, g\right)$ with the following properties:

(i) The differential equation

$$
\nabla_{X} \psi_{1}=\lambda p X \cdot \psi_{1}+\bar{\lambda} q \beta^{-1}(X) \cdot \psi_{1}
$$

holds for some constants $\lambda, \bar{\lambda} \in \mathbb{R}, \lambda \neq 0$, and for all vector fields $X$.

(ii) $\psi_{1}$ is a first eigenspinor of both $D$ and $\bar{D}$.

In the limiting case, the parameter $c=\bar{\lambda}_{1} / \lambda_{1}$ is the ratio of the two first eigenvalues.

Note that inequalities (1.1) and (1.3) both generalize Friedrich's inequality [5]

$$
\lambda_{1}^{2} \geq \frac{n}{4(n-1)} S_{\min }
$$

where $S_{\min }$ denotes the minimum of the (positive) scalar curvature. The limiting case of (1.5) is characterized by the existence of a Killing spinor $\psi_{1}$, i.e., a solution of the differential equation

$$
\nabla_{X} \psi_{1}=-\frac{\lambda}{n} X \cdot \psi_{1}, \quad \lambda \neq 0 \in \mathbb{R} .
$$

Any manifold admitting Killing spinors is necessarily Einstein and the simplyconnected manifolds admitting Killing spinors were completely classified $[2,3]$ : They are the standard spheres, 6-dimensional nearly Kähler nonKähler manifolds, Einstein Sasakian manifolds, 3-Sasakian manifolds and 7-dimensional manifolds with nearly parallel $G_{2}$-structure.

Let $():,=\operatorname{Re}\langle$,$\rangle denote the real part of the standard Hermitian product$ $\langle$,$\rangle on the spinor bundle \Sigma(M)$ over $M^{n}$. Let $\alpha=\alpha(\phi, \psi)$ be a 1 -form on $M^{n}$ induced by a nondegenerate symmetric tensor $\beta$ and spinor fields $\phi, \psi \in \Gamma(\Sigma)$ via

$$
\alpha(X)=\left(\phi, \beta^{-1}(X) \cdot \psi\right)
$$

Then

$$
\operatorname{div}(\alpha)=-\left(D_{\beta} \phi, \psi\right)+\left(\phi, D_{\beta} \psi\right)+\left(\phi, \operatorname{div}\left(\beta^{-1}\right) \cdot \psi\right) .
$$

Thus, if $\beta$ is a nondegenerate symmetric tensor with $\operatorname{div}\left(\beta^{-1}\right)=0$, then $D_{\beta}$ is a self-adjoint elliptic operator of first order and hence the spectrum of $D_{\beta}$ is discrete and real. 
In this paper we want to show that inequalities (1.1) and (1.3) remain valid even when we use a nondegenerate symmetric tensor $\beta$ with $\operatorname{div}\left(\beta^{-1}\right)=0$ to estimate lower bounds for the first eigenvalue of the Dirac operator. In our new theorems in the next section, Theorems 2.1 and $2.2, \bar{\lambda}_{1}$ shall denote the first eigenvalue of the operator $D_{\beta}$ and the free functions, $F=\left|\operatorname{det}\left(\beta^{-1}\right)\right| \cdot\left|\beta^{-1}\right|^{2}$ in (1.2) and $F=-\frac{\left|\operatorname{det}\left(\beta^{-1}\right)\right|}{q}$ in (1.4), will be replaced by $F=\left|\beta^{-1}\right|^{2}$ and $F=-\frac{1}{q}$, respectively. We will in fact transform inequality (1.3) into a more explicit form (2.15) and compare it with Friedrich's inequality (1.5). It turns out that inequality (2.15) can be sharper than (1.5) under certain additional conditions (see Remarks 2.2 and 3.3).

In the last section of the paper, we apply Theorems 2.1 and 2.2 to Sasakian spin manifolds and study the geometric meaning of the limiting case of the resulting inequalities (see Propositions 3.5 and 3.6). Recall that a Sasakian manifold $\left(M^{2 m+1}, \phi, \xi, \eta, g\right)$ of dimension $2 m+1$ consists of a $(1,1)$-tensor field $\phi$, a vector field $\xi$, a 1 -form $\eta$, and a metric $g$ that satisfy $\eta(\xi)=1$ and

$$
\begin{aligned}
& \phi^{2}(X)=-X+\eta(X) \xi, \quad g(\phi X, \phi Y)=g(X, Y)-\eta(X) \eta(Y), \\
& \left(\nabla_{X} \phi\right)(Y)=g(X, Y) \xi-\eta(Y) X
\end{aligned}
$$

for all vector fields $X, Y$. A Sasakian manifold $\left(M^{2 m+1}, \phi, \xi, \eta, g\right)$ is called eta-Einstein [4] if the Ricci curvature tensor Ric satisfies

$$
\mathrm{Ric}=\kappa g+\tau \eta \otimes \eta
$$

for some constants $\kappa, \tau \in \mathbb{R}$ with $\kappa+\tau=2 m$. Any eta-Einstein Sasakian manifold is necessarily of constant scalar curvature $S$ and we can rewrite etaEinstein condition (1.6) as

$$
\operatorname{Ric}=\left(\frac{S}{n-1}-1\right) g+\left(n-\frac{S}{n-1}\right) \eta \otimes \eta, \quad n=2 m+1 .
$$

Over Sasakian spin manifolds, a special class of spinors deserves attention [9].

Definition 1.2. A nontrivial spinor field $\psi$ on Sasakian spin manifold $\left(M^{2 m+1}\right.$, $\phi, \xi, \eta, g)$ is called an eta-Killing spinor with Killing pair $(a, b)$ if it satisfies

$$
\nabla_{X} \psi=a X \cdot \psi+b \eta(X) \xi \cdot \psi
$$

for some real numbers $a, b \in \mathbb{R}, a \neq 0$, and for all vector fields $X$.

In Propositions 3.1-3.4, we will establish some relations between the Killing pair $(a, b)$ of an eta-Killing spinor and the decomposition property of the spinor bundle over Sasakian manifolds, proving in particular that a simply connected closed Sasakian spin manifold is eta-Einstein if and only if there exists either an eta-Killing spinor with Killing pair

$$
\left(\frac{1}{2},-\frac{n}{4}+\frac{S}{4(n-1)}\right), \quad\left(-\frac{1}{2}, \frac{n}{4}-\frac{S}{4(n-1)}\right) \quad \text { for } n \geq 5
$$


or an eta-Killing spinor with Killing pair

$$
\left(\frac{-2+\sqrt{4+2 S}}{4}, \frac{4-\sqrt{4+2 S}}{4}\right) \text { for } n=3 \text {. }
$$

Applying Theorems 2.1 and 2.2 to divergencefree symmetric tensors $\beta^{-1}=$ $\frac{2}{n} I-2 \xi \otimes \eta$ and $\gamma^{-1}=I-2 \xi \otimes \eta$, we obtain Propositions 3.5 and 3.6, respectively. We will show that the limiting case of (3.9) occurs if and only if there exists an eta-Killing spinor with Killing pair (1.8) or (1.9) such that it is a first eigenspinor of $D_{\beta}$. Similarly, the limiting case of (3.14) is attained only if there exists an eta-Killing spinor with Killing pair (1.8) or (1.9) such that it is a first eigenspinor of $D_{\gamma}$.

\section{Dirac eigenvalues estimates in terms of divergencefree symmetric tensors}

We start with recalling some lemmata that we will need to prove Theorems 2.1 and 2.2 .

Lemma 2.1. Let $\beta$ be a nondegenerate symmetric tensor field on $\left(M^{n}, g\right)$. If there exists a nontrivial spinor field $\psi$ on $\left(M^{n}, g\right)$ such that

$$
\nabla_{X} \psi=p X \cdot D \psi+q \beta^{-1}(X) \cdot D_{\beta} \psi
$$

holds for some real-valued functions $p, q: M^{n} \longrightarrow \mathbb{R}$ and for all vector fields $X$, then we have

$$
(1+n p) D \psi=-q \operatorname{tr}\left(\beta^{-1}\right) D_{\beta} \psi, \quad\left(1+q\left|\beta^{-1}\right|^{2}\right) D_{\beta} \psi=-p \operatorname{tr}\left(\beta^{-1}\right) D \psi
$$

and

$$
\begin{aligned}
S \psi= & 4 d p \cdot D \psi+4 \beta^{-1}(d q) \cdot D_{\beta} \psi \\
& +4(p+1) D^{2} \psi+4 q D_{\beta}^{2} \psi+4 q \operatorname{div}\left(\beta^{-1}\right) \cdot D_{\beta} \psi
\end{aligned}
$$

Proof. Contracting both sides of (2.1) gives (2.2). Applying (2.1) to the formulas

$$
\begin{aligned}
\frac{1}{2} \operatorname{Ric}(X) \cdot \psi & =D\left(\nabla_{X} \psi\right)-\nabla_{X}(D \psi)-\sum_{i=1}^{n} E_{i} \cdot \nabla_{\nabla_{E_{i}} X} \psi \\
S \psi & =-\sum_{i=1}^{n} E_{i} \cdot \operatorname{Ric}\left(E_{i}\right) \cdot \psi
\end{aligned}
$$

we obtain

$$
\begin{aligned}
\frac{1}{2} \operatorname{Ric}(X) \cdot \psi= & \operatorname{grad}(p) \cdot X \cdot D \psi+\operatorname{grad}(q) \cdot \beta^{-1}(X) \cdot D_{\beta} \psi \\
& -p X \cdot D^{2} \psi-(2 p+1) \nabla_{X}(D \psi)-q \beta^{-1}(X) \cdot D\left(D_{\beta} \psi\right) \\
& -2 q \nabla_{\beta^{-1}(X)}\left(D_{\beta} \psi\right)+q \sum_{i=1}^{n} E_{i} \cdot\left(\nabla_{E_{i}} \beta^{-1}\right)(X) \cdot D_{\beta} \psi
\end{aligned}
$$

and identity (2.3), respectively. 
Lemma 2.2. Let $\psi$ and $F$ be a spinor field and a real-valued function on $\left(M^{n}, g\right)$, respectively. Then we have

$$
F \cdot \triangle(\psi, \psi)-(\psi, \psi) \cdot \triangle F=\operatorname{div}\{(\psi, \psi) \operatorname{grad} F-F \operatorname{grad}(\psi, \psi)\} .
$$

Theorem 2.1. Let $\left(M^{n}, g\right)$ be an $n$-dimensional closed Riemannian spin manifold. Let $\beta$ be such a nondegenerate symmetric tensor on $M^{n}$ that both $\operatorname{div}\left(\beta^{-1}\right)$ $=0$ and $\operatorname{tr}\left(\beta^{-1}\right)=0$ vanish identically. Let $\lambda_{1} \in \mathbb{R}$ and $\bar{\lambda}_{1} \in \mathbb{R}$ be the first eigenvalue of $D$ and $D_{\beta}$, respectively. Then we have

$$
\lambda_{1}^{2} \geq \inf _{M}\left\{\frac{n S}{4(n-1)}+\frac{n \bar{\lambda}_{1}^{2}}{(n-1)\left|\beta^{-1}\right|^{2}}+\frac{n \Delta F}{2(n-1) F}\right\},
$$

where $F: M^{n} \longrightarrow \mathbb{R}$ is a real-valued function defined by $F=\left|\beta^{-1}\right|^{2}$.

The limiting case of (2.5) occurs if and only if $\triangle F=0$ vanishes identically and there exists a spinor field $\psi_{1}$ on $\left(M^{n}, g\right)$ with the following properties:

(i) The differential equation

$$
\nabla_{X} \psi_{1}=-\frac{\lambda}{n} X \cdot \psi_{1}-\frac{\bar{\lambda}}{\left|\beta^{-1}\right|^{2}} \beta^{-1}(X) \cdot \psi_{1}
$$

holds for some constants $\lambda, \bar{\lambda} \in \mathbb{R}$ and for all vector fields $X$.

(ii) $\psi_{1}$ is a first eigenspinor of both $D$ and $D_{\beta}$.

Proof. Let $\mathcal{Q}: \Gamma(T(M)) \times \Gamma\left(\Sigma(M)_{g}\right) \longrightarrow \Gamma\left(\Sigma(M)_{g}\right)$ be a twistor-like operator defined by

$$
\mathcal{Q}_{X}(\varphi)=\nabla_{X} \varphi-p X \cdot D \varphi-q \beta^{-1}(X) \cdot D_{\beta} \varphi
$$

where $p, q: M^{n} \longrightarrow \mathbb{R}$ are some real-valued functions. Then we have

$$
\begin{aligned}
& \sum_{j=1}^{n}\left(\mathcal{Q}_{E_{j}}(\varphi), \mathcal{Q}_{E_{j}}(\varphi)\right) \\
&= \operatorname{div}\left[\sum_{j=1}^{n}\left(\varphi, E_{j} \cdot D \varphi+\nabla_{E_{j}} \varphi\right) E_{j}\right]+\left(n p^{2}+2 p+1\right)(D \varphi, D \varphi)-\frac{1}{4} S(\varphi, \varphi) \\
&(2.7) \quad+\left\{q^{2}\left|\beta^{-1}\right|^{2}+2 q\right\}\left(D_{\beta} \varphi, D_{\beta} \varphi\right)+2 p q \operatorname{tr}\left(\beta^{-1}\right)\left(D \varphi, D_{\beta} \varphi\right) .
\end{aligned}
$$

Let $\psi_{1}$ be an eigenspinor of $D$ for the first eigenvalue $\lambda_{1}$. Let $\mu$ denote the volume form of $\left(M^{n}, g\right)$. By Lemma 2.2, we then see that

$$
\int_{M^{n}} F \operatorname{div}\left[\sum_{j=1}^{n}\left(\psi_{1}, E_{j} \cdot D \psi_{1}+\nabla_{E_{j}} \psi_{1}\right) E_{j}\right] \mu=-\frac{1}{2} \int_{M^{n}}\left(\psi_{1}, \psi_{1}\right) \triangle(F) \mu
$$

holds for any real-valued function $F: M^{n} \longrightarrow \mathbb{R}$, since $\left(\psi_{1}, E_{j} \cdot \psi_{1}\right)=0$ vanishes identically. Now, let $\bar{\lambda}_{1}$ be the first eigenvalue of $D_{\beta}$. Making use of (2.7)-(2.8) and $\operatorname{tr}\left(\beta^{-1}\right)=0$ and introducing a free function $F: M^{n} \longrightarrow \mathbb{R}$, 
which is assumed to be a positive function, to control the unnecessary terms, we compute

$$
\begin{aligned}
H_{1}:= & \int_{M^{n}}\left[\left(D_{\beta} \psi_{1}, D_{\beta} \psi_{1}\right)-\bar{\lambda}_{1}^{2}\left(\psi_{1}, \psi_{1}\right)\right] \mu+\int_{M^{n}}\left[F \sum_{j=1}^{n}\left(\mathcal{Q}_{E_{j}}\left(\psi_{1}\right), \mathcal{Q}_{E_{j}}\left(\psi_{1}\right)\right)\right] \mu \\
= & \int_{M^{n}}\left[\lambda_{1}^{2}\left(n p^{2}+2 p+1\right) F-\frac{1}{4} F S-\bar{\lambda}_{1}^{2}-\frac{1}{2}(\triangle F)\right]\left(\psi_{1}, \psi_{1}\right) \mu \\
& +\int_{M^{n}}\left[\left(q^{2}\left|\beta^{-1}\right|^{2}+2 q\right) F+1\right]\left(D_{\beta} \psi_{1}, D_{\beta} \psi_{1}\right) \mu .
\end{aligned}
$$

Choose the free parameters $p, q, F$ as

$$
p=-\frac{1}{n}, \quad q=-\frac{1}{\left|\beta^{-1}\right|^{2}}, \quad F=\left|\beta^{-1}\right|^{2} .
$$

Then the last line of (2.9) vanishes and we have

$$
H_{1}=\int_{M^{n}}\left[\frac{(n-1) \lambda_{1}^{2} F}{n}-\frac{1}{4} F S-\bar{\lambda}_{1}^{2}-\frac{1}{2}(\triangle F)\right]\left(\psi_{1}, \psi_{1}\right) \mu \geq 0,
$$

which proves inequality (2.5). The conditions (i)-(ii) for the limiting case of (2.5) are clear.

Assume that $\lambda_{1} \neq 0$ is nonzero, and let $c_{1}:=\bar{\lambda}_{1} / \lambda_{1}$ denote the ratio of the two first eigenvalues. Then (2.10) can be rewritten as

$$
H_{2}=\int_{M^{n}}\left[\lambda_{1}^{2} F\left(\frac{n-1}{n}-\frac{c_{1}^{2}}{\left|\beta^{-1}\right|^{2}}\right)-\frac{1}{4} F S-\frac{1}{2}(\triangle F)\right]\left(\psi_{1}, \psi_{1}\right) \mu \geq 0
$$

This proves:

Corollary 2.1. In the notations of Theorem 2.1, we have

$$
\lambda_{1}^{2} \geq \inf _{M_{*}}\left\{\frac{S}{4 \rho}+\frac{\triangle F}{2 \rho F}\right\}
$$

where $\rho$ is a real-valued function on $M^{n}$ defined by

$$
\rho=\frac{n-1}{n}-\frac{c_{1}^{2}}{\left|\beta^{-1}\right|^{2}}
$$

and the infimum is taken over the subset $M_{*} \subset M^{n}$,

$$
M_{*}:=\left\{x \in M^{n}: \rho(x)>0\right\} .
$$

Remark 2.1. If the scalar curvature $S>0$ is positive and $\left|\beta^{-1}\right|^{2}$ is constant on $M^{n}$, then (2.11) implies that $\rho>0$ is a positive constant and inequality (2.12) is sharper than Friedrich's inequality (1.5). In fact, (2.12) reduces to (1.5) if $c_{1}=0$ equals zero and $\left|\beta^{-1}\right|^{2}$ is a constant. 
Theorem 2.2. Let $\left(M^{n}, g\right)$ be an $n$-dimensional closed Riemannian spin manifold. Let $\beta$ be a nondegenerate symmetric tensor on $M^{n}$ such that $\operatorname{div}\left(\beta^{-1}\right)=0$ and $n\left|\beta^{-1}\right|^{2}>\left(\operatorname{tr} \beta^{-1}\right)^{2}>0$ hold at all points. Let $\lambda_{1} \neq 0 \in \mathbb{R}$ and $\bar{\lambda}_{1} \in \mathbb{R}$ be the first eigenvalue of $D$ and $D_{\beta}$, respectively, and denote $c_{1}=\bar{\lambda}_{1} / \lambda_{1}$. For any real-valued function $C: M^{n} \longrightarrow \mathbb{R}$ satisfying either

$$
\frac{\operatorname{tr}\left(\beta^{-1}\right)}{n}<C<\frac{\left|\beta^{-1}\right|^{2}}{\operatorname{tr}\left(\beta^{-1}\right)}, \quad \operatorname{tr}\left(\beta^{-1}\right)>0,
$$

or

$$
\frac{\operatorname{tr}\left(\beta^{-1}\right)}{n}>C>\frac{\left|\beta^{-1}\right|^{2}}{\operatorname{tr}\left(\beta^{-1}\right)}, \quad \operatorname{tr}\left(\beta^{-1}\right)<0
$$

we have

$$
\lambda_{1}^{2} \geq \inf _{M_{*}}\left\{\frac{S}{4 \rho(C)}+\frac{\triangle F}{2 \rho(C) F}\right\},
$$

where $\rho(C)$ and $F$ are real-valued functions on $M^{n}$ defined by

$$
\begin{aligned}
\rho(C) & =\frac{\left(\operatorname{tr} \beta^{-1}\right) C^{2}+\left[(n-1)\left|\beta^{-1}\right|^{2}-\left(\operatorname{tr} \beta^{-1}\right)^{2}-n c_{1}^{2}\right] C+\left(\operatorname{tr} \beta^{-1}\right) c_{1}^{2}}{C\left[n\left|\beta^{-1}\right|^{2}-\left(\operatorname{tr} \beta^{-1}\right)^{2}\right]}, \\
F & =\frac{C\left[n\left|\beta^{-1}\right|^{2}-\left(\operatorname{tr} \beta^{-1}\right)^{2}\right]}{C n-\operatorname{tr}\left(\beta^{-1}\right)}
\end{aligned}
$$

respectively, and $M_{*}=\{x \in M: \rho(C)(x)>0\}$.

The limiting case of (2.15) occurs if and only if $\triangle F=0$ vanishes identically, $C=c_{1}=\bar{\lambda}_{1} / \lambda_{1}$ is a constant and there exists a spinor field $\psi_{1}$ on $\left(M^{n}, g\right)$ with the following properties:

(i) The differential equation

$$
\nabla_{X} \psi_{1}=\lambda p X \cdot \psi_{1}+\bar{\lambda} q \beta^{-1}(X) \cdot \psi_{1}
$$

holds for some real-valued functions $p, q$ on $M^{n}$, for some constants $\lambda, \bar{\lambda} \in \mathbb{R}$, $\lambda \neq 0$, and for all vector fields $X$.

(ii) $\psi_{1}$ is a first eigenspinor of both $D$ and $D_{\beta}$.

In the limiting case, the functions $p$ and $q$ are expressed as (2.22) with $C=$ $c_{1}=\bar{\lambda}_{1} / \lambda_{1}$.

Proof. Let $\mathcal{Q}: \Gamma(T(M)) \times \Gamma\left(\Sigma(M)_{g}\right) \longrightarrow \Gamma\left(\Sigma(M)_{g}\right)$ be a twistor-like operator defined by

$$
\mathcal{Q}_{X}(\varphi)=\nabla_{X} \varphi-p X \cdot D \varphi-q \beta^{-1}(X) \cdot D_{\beta} \varphi,
$$

where $p, q: M^{n} \longrightarrow \mathbb{R}$ are some real-valued functions. Let $\psi_{1}$ be an eigenspinor of $D$ for the first eigenvalue $\lambda_{1} \neq 0$. Let $\bar{\lambda}_{1}=c_{1} \lambda_{1}$ be the first eigenvalue of $D_{\beta}$. Making use of (2.7)-(2.8) and introducing free functions $F, B, C: M^{n} \longrightarrow \mathbb{R}$ 
(We assume that $F$ is a positive function.) to control the unnecessary terms, we compute

$$
\begin{aligned}
H_{2}:= & \int_{M^{n}}\left[\left(D_{\beta} \psi_{1}, D_{\beta} \psi_{1}\right)-c_{1}^{2} \lambda_{1}^{2}\left(\psi_{1}, \psi_{1}\right)\right] \mu \\
& +\int_{M^{n}}\left[F \sum_{j=1}^{n}\left(\mathcal{Q}_{E_{j}}\left(\psi_{1}\right), \mathcal{Q}_{E_{j}}\left(\psi_{1}\right)\right)+B^{2}\left(D_{\beta} \psi_{1}-C D \psi_{1}, D_{\beta} \psi_{1}-C D \psi_{1}\right)\right] \mu \\
= & \int_{M^{n}}\left[\lambda_{1}^{2}\left(\left(n p^{2}+2 p+1\right) F+B^{2} C^{2}-c_{1}^{2}\right)-\frac{1}{4} F S-\frac{1}{2}(\triangle F)\right]\left(\psi_{1}, \psi_{1}\right) \mu \\
& +\int_{M^{n}}\left[2 \lambda_{1}\left(p q F \operatorname{tr}\left(\beta^{-1}\right)-B^{2} C\right)\left(\psi_{1}, D_{\beta} \psi_{1}\right)\right. \\
& \left.+\left(\left(q^{2}\left|\beta^{-1}\right|^{2}+2 q\right) F+B^{2}+1\right)\left(D_{\beta} \psi_{1}, D_{\beta} \psi_{1}\right)\right] \mu .
\end{aligned}
$$

We choose the functions $F, B, C$ in such a way that the last two lines of (2.17) vanish and the equations in (2.2) are satisfied with $D_{\beta} \psi_{1}=C D \psi_{1}$. Namely, the functions $p, q, B, F$ have to satisfy the following four equations:

$$
\begin{aligned}
& n p+C\left(\operatorname{tr} \beta^{-1}\right) q=-1, \\
& \left(\operatorname{tr} \beta^{-1}\right) p+C\left|\beta^{-1}\right|^{2} q=-C, \\
& p q F\left(\operatorname{tr} \beta^{-1}\right)-B^{2} C=0, \\
& \left(q^{2}\left|\beta^{-1}\right|^{2}+2 q\right) F+B^{2}+1=0 .
\end{aligned}
$$

Solving (2.18)-(2.21), we obtain

$$
p=\frac{-\left|\beta^{-1}\right|^{2}+C\left(\operatorname{tr} \beta^{-1}\right)}{n\left|\beta^{-1}\right|^{2}-\left(\operatorname{tr} \beta^{-1}\right)^{2}}, \quad q=\frac{-C n+\left(\operatorname{tr} \beta^{-1}\right)}{C\left[n\left|\beta^{-1}\right|^{2}-\left(\operatorname{tr} \beta^{-1}\right)^{2}\right]},
$$

and

$$
\begin{aligned}
F & =-\frac{1}{q}=\frac{C\left[n\left|\beta^{-1}\right|^{2}-\left(\operatorname{tr} \beta^{-1}\right)^{2}\right]}{C n-\operatorname{tr}\left(\beta^{-1}\right)}, \\
B^{2} & =1+q\left|\beta^{-1}\right|^{2}=\frac{-C\left(\operatorname{tr} \beta^{-1}\right)^{2}+\left|\beta^{-1}\right|^{2}\left(\operatorname{tr} \beta^{-1}\right)}{C\left[n\left|\beta^{-1}\right|^{2}-\left(\operatorname{tr} \beta^{-1}\right)^{2}\right]} .
\end{aligned}
$$

Note that (2.18) and (2.19) together give

and so the relations

$$
C=-\frac{1+n p}{q\left(\operatorname{tr} \beta^{-1}\right)}=-\frac{p\left(\operatorname{tr} \beta^{-1}\right)}{1+q\left|\beta^{-1}\right|^{2}}
$$

$$
F=-\frac{1}{q}>0, \quad B^{2}=1+q\left|\beta^{-1}\right|^{2}>0, \quad C^{2}=\frac{p(1+n p)}{q\left(1+q\left|\beta^{-1}\right|^{2}\right)}>0
$$


imply the restriction

$$
-\frac{1}{\left|\beta^{-1}\right|^{2}}<q<0, \quad-\frac{1}{n}<p<0
$$

It is easy to check, using the relations in (2.22), that (2.26) can equivalently be expressed as (2.13)-(2.14). Now, inserting (2.23)-(2.24) into (2.17), we have

$$
H_{2}=\int_{M^{n}}\left[\lambda_{1}^{2}\left(p+1+c_{1}^{2} q\right) F-\frac{1}{4} F S-\frac{1}{2}(\triangle F)\right]\left(\psi_{1}, \psi_{1}\right) \mu \geq 0,
$$

and applying (2.22) to (2.27) proves inequality (2.15), where $\rho(C):=p+1+c_{1}^{2} q$. The conditions (i)-(ii) for the limiting case of (2.15) are easy to check.

Remark 2.2. Assume that, in Theorem 2.2, the scalar curvature $S>0$ is positive and both $\operatorname{tr}\left(\beta^{-1}\right)$ and $\left|\beta^{-1}\right|^{2}$ are positive constants. Choose a constant $C=c$ satisfying (2.13). Then (2.27) implies that $\rho(C=c)>0$ is a positive constant. Therefore, if we can choose a constant $c$ such that

$$
\left[n c-\operatorname{tr}\left(\beta^{-1}\right)\right]\left[\left(\operatorname{tr} \beta^{-1}\right) c-n c_{1}^{2}\right]<0
$$

is satisfied(see Remark 3.3), then (2.15) is sharper than Friedrich's inequality (1.5), for a simple computation gives

$$
\frac{1}{\rho(c)}-\frac{n}{n-1}=-\frac{\left[n c-\operatorname{tr}\left(\beta^{-1}\right)\right]\left[\left(\operatorname{tr} \beta^{-1}\right) c-n c_{1}^{2}\right]}{c(n-1)\left[n\left|\beta^{-1}\right|^{2}-\left(\operatorname{tr} \beta^{-1}\right)^{2}\right] \rho(c)}>0 .
$$

Remark 2.3. Inequality (2.15) remains essentially the same when the tensor $\beta$ with $\operatorname{div}\left(\beta^{-1}\right)=0$ is replaced by a Codazzi tensor $\beta$ (see Theorem B in Section $1)$. In this case, $\bar{\lambda}_{1}$ should denote the first eigenvalue of the Dirac operator $\bar{D}$ of $\bar{g}(\cdot, \cdot):=g(\beta(\cdot), \beta(\cdot))$ and the free function $F=-\frac{1}{q}$ in $(2.23)$ must be replaced by

$$
F=-\frac{\left|\operatorname{det}\left(\beta^{-1}\right)\right|}{q}=\frac{C\left|\operatorname{det}\left(\beta^{-1}\right)\right|\left[n\left|\beta^{-1}\right|^{2}-\left(\operatorname{tr} \beta^{-1}\right)^{2}\right]}{C n-\operatorname{tr}\left(\beta^{-1}\right)} .
$$

Remark 2.4. Assume that, in Theorem 2.1, $\left|\beta^{-1}\right|^{2}$ is constant on $M^{n}$. Let $\psi_{1}$ be a spinor field on $\left(M^{n}, g\right)$ satisfying equation (2.6) and suppose that $\psi_{1}$ is a first eigenspinor of $D_{\beta}$, i.e., $\bar{\lambda}=\bar{\lambda}_{1}$. Then, by (2.3), we have

$$
\lambda^{2}=\frac{n S}{4(n-1)}+\frac{n \bar{\lambda}_{1}^{2}}{(n-1)\left|\beta^{-1}\right|^{2}} .
$$

Comparing (2.28) to (2.5), we conclude that $\psi_{1}$ is also a first eigenspinor of $D$. Similarly, when, in Theorem 2.2 , both $\operatorname{tr}\left(\beta^{-1}\right)$ and $\left|\beta^{-1}\right|^{2}$ are constant on $M^{n}$, the second condition "(ii) $\psi_{1}$ is a first eigenspinor of both $D$ and $D_{\beta}$ " for the limiting case of (2.15) simplifies to "(ii) $\psi_{1}$ is a first eigenspinor of $D_{\beta}$ ". 


\section{Dirac eigenvalues estimates over Sasakian manifolds}

In this section we will apply Theorems 2.1 and 2.2 to Sasakian spin manifolds and investigate the geometric implication of the limiting case of the resulting inequalities (see Propositions 3.5 and 3.6). For this purpose we need to establish some relations between the Killing pair $(a, b)$ of an eta-Killing spinor(see Definition 1.2) and the decomposition property of the spinor bundle over Sasakian manifolds.

Consider a manifold $M^{2 m+1}$ of odd dimension $2 m+1$. An almost contact metric structure $(\phi, \xi, \eta, g)$ of $M^{2 m+1}$ consists of a (1,1)-tensor field $\phi$, a vector field $\xi$, a 1-form $\eta$, and a metric $g$ with the following properties:

$$
\eta(\xi)=1, \quad \phi^{2}(X)=-X+\eta(X) \xi, \quad g(\phi X, \phi Y)=g(X, Y)-\eta(X) \eta(Y) .
$$

The fundamental 2-form $\Phi$ of the contact structure is a 2 -form defined by

$$
\Phi(X, Y)=g(X, \phi(Y)) .
$$

An almost contact metric structure $(\phi, \xi, \eta, g)$ of $M^{2 m+1}$ is called Sasakian structure if

$$
\left(\nabla_{X} \phi\right)(Y)=g(X, Y) \xi-\eta(Y) X
$$

holds for all vector fields $X, Y$. The Riemann curvature tensor $R$ of Sasakian manifold $\left(M^{2 m+1}, \phi, \xi, \eta, g\right)$ has some special symmetries, i.e.,

$$
\begin{aligned}
g(R(\phi X, \phi Y)(\phi Z), \phi W)= & g(R(X, Y) Z, W)+\eta(Y) \eta(W) g(X, Z) \\
& -\eta(Y) \eta(Z) g(X, W)-\eta(X) \eta(W) g(Y, Z) \\
& +\eta(X) \eta(Z) g(Y, W) .
\end{aligned}
$$

Expressing the components of the curvature tensor $R$ with respect to an adapted local orthonormal frame $E_{1}, E_{\overline{1}}:=\phi\left(E_{1}\right), E_{2}, E_{\overline{2}}:=\phi\left(E_{2}\right), \ldots, E_{\bar{m}}:=\phi\left(E_{m}\right)$, $E_{2 m+1}:=\xi$, we can reformulate identity (3.1) as follows.

Lemma 3.1 ([9]). On any Sasakian manifold $\left(M^{2 m+1}, \phi, \xi, \eta, g\right)$, we have

$$
\begin{aligned}
& R_{\bar{i} \bar{j} \bar{k} \bar{l}}=R_{i j k l}, \quad R_{i j \bar{k} \bar{l}}=R_{\bar{i} \bar{j} k l}, \quad R_{i \bar{j} k \bar{l}}=R_{\bar{i} j \bar{k} l}, \\
& R_{i \bar{j} \bar{k} \bar{l}}=-R_{\bar{i} j k l}, \quad R_{\bar{i} \bar{j} k \bar{l}}=-R_{i j \bar{k} l}, \\
& R_{i 2 m+1 k 2 m+1}=R_{\bar{i} 2 m+1 \bar{k} 2 m+1}=\delta_{i k} \quad(1 \leq i, j, k, l \leq m) .
\end{aligned}
$$

In all the other cases, $R_{u v w z}=0$ as soon as one of its indices equals $2 m+1$.

Assume that an almost contact metric manifold $\left(M^{2 m+1}, \phi, \xi, \eta, g\right)$ has a spin structure. Then the spinor bundle of $\left(M^{2 m+1}, \phi, \xi, \eta, g\right)$ splits under the action of the fundamental 2-form $\Phi$ as follows.

Lemma $3.2([9])$. Let $\left(M^{2 m+1}, \phi, \xi, \eta, g\right)$ be an almost contact metric manifold with spin structure and fundamental 2 -form $\Phi$. Then the spinor bundle $\Sigma$ splits into the orthogonal direct sum $\Sigma=\Sigma_{0} \oplus \Sigma_{1} \oplus \cdots \oplus \Sigma_{m}$ with

(i) $\left.\Phi\right|_{\Sigma_{r}}=\sqrt{-1}(2 r-m) I, \quad \operatorname{dim}\left(\Sigma_{r}\right)=\left(\begin{array}{c}m \\ r\end{array}\right) \quad(0 \leq r \leq m)$, 
(ii) $\left.\xi\right|_{\Sigma_{0} \oplus \Sigma_{2} \oplus \Sigma_{4} \oplus \cdots}=(\sqrt{-1})^{2 m+1} I,\left.\quad \xi\right|_{\Sigma_{1} \oplus \Sigma_{3} \oplus \Sigma_{5} \oplus \cdots}=-(\sqrt{-1})^{2 m+1} I$, where I stands for the identity map. Moreover, the bundles $\Sigma_{0}$ and $\Sigma_{m}$ can be defined by

$\Sigma_{0}=\left\{\psi \in \Sigma: \phi(X) \cdot \psi+\sqrt{-1} X \cdot \psi+(-1)^{m} \eta(X) \psi=0\right.$ for all vectors $\left.X\right\}$, $\Sigma_{m}=\{\psi \in \Sigma: \phi(X) \cdot \psi-\sqrt{-1} X \cdot \psi-\eta(X) \psi=0$ for all vectors $X\}$.

In particular, we have the formulas

$$
\begin{aligned}
& \xi \cdot \psi_{0}=(-1)^{m} \sqrt{-1} \psi_{0}, \quad \Phi \cdot \psi_{0}=-m \sqrt{-1} \psi_{0}, \quad \psi_{0} \in \Sigma_{0}, \\
& \xi \cdot \psi_{m}=\sqrt{-1} \psi_{m}, \quad \Phi \cdot \psi_{m}=m \sqrt{-1} \psi_{m}, \quad \psi_{m} \in \Sigma_{m} .
\end{aligned}
$$

Obviously, any eta-Killing spinor with Killing pair $(a, b)$ is an eigenspinor of the Dirac operator with eigenvalue $\lambda=-(2 m+1) a-b$. Now we further establish some basic relations between the Killing pair $(a, b)$ of an eta-Killing spinor and the geometry of the Sasakian manifold. For proofs for Propositions 3.2-3.4 we refer to [9]. In the following we will often write $n$ to mean the dimension $2 m+1$ of the manifold $M^{2 m+1}$.

Proposition 3.1. Let $\left(M^{2 m+1}, \phi, \xi, \eta, g\right), m \geq 2$, be a Sasakian spin manifold and suppose that it admits an eta-Killing spinor $\psi$ with Killing pair $(a, b)$, where both $a \neq 0$ and $b \neq 0$ are nonzero. Then $\left(M^{2 m+1}, \phi, \xi, \eta, g\right)$ is eta-Einstein with scalar curvature $S=4 n(n-1) a^{2}+8(n-1) a b$. Moreover, all the possible values for $a, b$ can be expressed in terms of the scalar curvature as

$$
(a, b)=\left(\frac{1}{2},-\frac{n}{4}+\frac{S}{4(n-1)}\right), \quad\left(-\frac{1}{2}, \frac{n}{4}-\frac{S}{4(n-1)}\right),
$$

and the following statements are true:

(i) If $(a, b)=\left(\frac{1}{2},-\frac{n}{4}+\frac{S}{4(n-1)}\right)$, then $m \equiv 0(\bmod 2)$ and $\psi \in \Gamma\left(\Sigma_{0}\right)$ is a section in $\Sigma_{0}$.

(ii) If $(a, b)=\left(-\frac{1}{2}, \frac{n}{4}-\frac{S}{4(n-1)}\right)$ and $m \equiv 0(\bmod 2)$, then $\psi \in \Gamma\left(\Sigma_{m}\right)$ is a section in $\Sigma_{m}$.

(iii) If $(a, b)=\left(-\frac{1}{2}, \frac{n}{4}-\frac{S}{4(n-1)}\right)$ and $m \equiv 1(\bmod 2)$, then $\psi \in \Gamma\left(\Sigma_{0}\right) \cup$ $\Gamma\left(\Sigma_{m}\right)$ is a section in $\Sigma_{0}$ or in $\Sigma_{m}$.

Proof. Let $\psi$ be an eta-Killing spinor with Killing pair $(a, b)$, where $a$ and $b$ both are nonzero. On the one hand, a direct computation gives

$$
\begin{aligned}
R\left(E_{k}, \xi\right)(\psi) & =\nabla_{E_{k}} \nabla_{\xi} \psi-\nabla_{\xi} \nabla_{E_{k}} \psi-\nabla_{\left[E_{k}, \xi\right]} \psi \\
& =-b E_{\bar{k}} \cdot \psi-2 a(a+b) E_{k} \cdot \xi \cdot \psi
\end{aligned}
$$

On the other hand, applying Lemma 3.1 to

$$
R\left(E_{k}, \xi\right)(\psi)=-\frac{1}{2} R\left(E_{k}, \xi\right) \cdot \psi=-\frac{1}{2} \sum_{u<v} R\left(E_{u}, E_{v}, E_{k}, \xi\right) E_{u} \cdot E_{v} \cdot \psi
$$


gives

$$
R\left(E_{k}, \xi\right)(\psi)=-\frac{1}{2} E_{k} \cdot \xi \cdot \psi
$$

Thus, combining (3.2) and (3.3), we obtain

$$
\{4 a(a+b)-1\} E_{k} \cdot \xi \cdot \psi+2 b E_{\bar{k}} \cdot \psi=0, \quad 1 \leq k \leq m .
$$

Since (3.4) implies

$$
\left(\{4 a(a+b)-1\} E_{k} \cdot \xi \cdot \psi,\{4 a(a+b)-1\} E_{k} \cdot \xi \cdot \psi\right)=\left(2 b E_{\bar{k}} \cdot \psi, 2 b E_{\bar{k}} \cdot \psi\right),
$$

we have

$$
\begin{aligned}
& \{4 a(a+b)-1\}^{2}=4 b^{2} \\
\Longleftrightarrow & (2 a-1)(2 a+1)(2 a+2 b-1)(2 a+2 b+1)=0 \\
\Longleftrightarrow & a= \pm \frac{1}{2} \quad \text { or } \quad a+b= \pm \frac{1}{2} .
\end{aligned}
$$

Using the decomposition properties in Lemma 3.2, we now show that the latter case $a+b= \pm \frac{1}{2}$ of (3.5) is not allowed. Comparing (3.4)-(3.5) to the definitions of the bundles $\Sigma_{0}$ and $\Sigma_{m}$ in Lemma 3.2, we find that $\psi \in \Gamma\left(\Sigma_{0}\right) \cup \Gamma\left(\Sigma_{m}\right)$ must be a section in $\Sigma_{0}$ or in $\Sigma_{m}$. Suppose first that $\psi \in \Gamma\left(\Sigma_{0}\right)$. Then, since $\left(\nabla_{X} \Phi\right) \cdot \psi=-X \cdot \xi \cdot \psi-\eta(X) \psi$, we have

$$
\begin{aligned}
& \Phi \cdot \nabla_{X} \psi=\nabla_{X}(\Phi \cdot \psi)-\left(\nabla_{X} \Phi\right) \cdot \psi \\
= & -m \sqrt{-1} \nabla_{X} \psi+(-1)^{m} \sqrt{-1} X \cdot \psi+\eta(X) \psi .
\end{aligned}
$$

Inserting $\nabla_{X} \psi=a X \cdot \psi+b \eta(X) \xi \cdot \psi$ into both sides of (3.6) and using the identity $X \cdot \Phi \cdot \psi-\Phi \cdot X \cdot \psi=2 \phi(X) \cdot \psi$, we come to

$$
\begin{aligned}
& (-a m+2 a) \sqrt{-1} X \cdot \psi+(-1)^{m}(2 a+b m) \eta(X) \psi \\
= & {\left[-a m+(-1)^{m}\right] \sqrt{-1} X \cdot \psi+\left[1+(-1)^{m} b m\right] \eta(X) \psi, }
\end{aligned}
$$

which gives $a=(-1)^{m} \frac{1}{2}$. Similarly, we conclude in the second case $\psi \in \Gamma\left(\Sigma_{m}\right)$ that $a=-\frac{1}{2}$. To prove that $\left(M^{2 m+1}, \phi, \xi, \eta, g\right)$ is eta-Einstein, it suffices to compute the Ricci tensor

$$
\begin{gathered}
\frac{1}{2} \operatorname{Ric}(X) \cdot \psi=D\left(\nabla_{X} \psi\right)-\nabla_{X}(D \psi)-\sum_{u=1}^{2 m+1} E_{u} \cdot \nabla_{\nabla_{E_{u}} X} \psi \\
=\left(4 m a^{2}+2 a b\right) X \cdot \psi+b \phi(X) \cdot \xi \cdot \psi+\left(m-4 m a^{2}-2 a b\right) \eta(X) \xi \cdot \psi
\end{gathered}
$$

and apply (3.4)-(3.5) to (3.7). Finally, contracting both sides of (3.7), we obtain

$$
S=4 n(n-1) a^{2}+8(n-1) a b=n(n-1) \pm 4(n-1) b,
$$

which enables us to express the Killing pair $(a, b)$ in terms of the scalar curvature. 
Remark 3.1. Following the arguments in the proof of Proposition 3.1, one proves easily that any Sasakian spin manifold $\left(M^{2 m+1}, \phi, \xi, \eta, g\right), m \geq 2$, admits no eta-Killing spinors with Killing pair $(0, b), b \neq 0 \in \mathbb{R}$. However, there exists an eta-Killing spinor with Killing pair (0, $\left.\frac{1}{2}\right)$ over every 3-dimensional simply-connected eta-Einstein Sasakian spin manifold (see Proposition 3.4). Note further that there exist no parallel spinors over Sasakian manifolds, since Sasakian manifolds cannot be Ricci-flat.

It was proven in [9] that, for simply-connected Sasakian spin manifolds, the converse of Proposition 3.1 holds.

Proposition $3.2([9])$. Let $\left(M^{2 m+1}, \phi, \xi, \eta, g\right), m \geq 2$, be a simply-connected Sasakian spin manifold. Suppose that $\left(M^{2 m+1}, \phi, \xi, \eta, g\right)$ is eta-Einstein. Then, in case

(i) $m \equiv 0(\bmod 2)$, there exists an eta-Killing spinor $\psi_{0} \in \Gamma\left(\Sigma_{0}\right)$ with Killing pair $\left(\frac{1}{2},-\frac{n}{4}+\frac{S}{4(n-1)}\right)$ as well as an eta-Killing spinor $\psi_{m} \in \Gamma\left(\Sigma_{m}\right)$ with Killing pair $\left(-\frac{1}{2}, \frac{n}{4}-\frac{S}{4(n-1)}\right)$.

(ii) $m \equiv 1(\bmod 2)$, there exist two eta-Killing spinors $\psi_{0}, \psi_{m}$ with Killing pair $\left(-\frac{1}{2}, \frac{n}{4}-\frac{S}{4(n-1)}\right)$ such that $\psi_{\alpha}$ is a section in the bundle $\Sigma_{\alpha}(\alpha=0, m)$.

Over 3-dimensional Sasakian spin manifolds $\left(M^{3}, \phi, \xi, \eta, g\right)$, relation (3.4) implies

$$
a=-\frac{1}{2} \quad \text { or } \quad a+b=\frac{1}{2}
$$

In contrast to higher dimensional case $m \geq 2$, the latter relation $a+b=\frac{1}{2}$ is allowed in 3-dimensional case, since $-E_{1} \cdot \xi \cdot \psi+E_{\overline{1}} \cdot \psi=0$ holds for all spinor fields $\psi \in \Gamma\left(\Sigma=\Sigma_{0} \oplus \Sigma_{1}\right)$.

Proposition $3.3([9])$. Let $\left(M^{3}, \phi, \xi, \eta, g\right)$ be a 3-dimensional Sasakian spin manifold and suppose that it admits an eta-Killing spinor $\psi$ with Killing pair $(a, b)$, where $a \neq 0$ and $b \neq 0$. Then $\left(M^{3}, \phi, \xi, \eta, g\right)$ is eta-Einstein with constant scalar curvature $S=24 a^{2}+16 a b$. Moreover, all the possible values for $a, b$ can be expressed in terms of the scalar curvature as

$$
\begin{aligned}
(a, b)= & \left(-\frac{1}{2}, \frac{3}{4}-\frac{S}{8}\right),\left(\frac{-2+\sqrt{4+2 S}}{4}, \frac{4-\sqrt{4+2 S}}{4}\right), \\
& \left(\frac{-2-\sqrt{4+2 S}}{4}, \frac{4+\sqrt{4+2 S}}{4}\right) .
\end{aligned}
$$

To state the converse of Proposition 3.3, we note that any 3-dimensional Sasakian manifold with constant scalar curvature is eta-Einstein in the sense of (1.6).

Proposition $3.4([9])$. Let $\left(M^{3}, \phi, \xi, \eta, g\right)$ be a simply-connected Sasakian spin manifold of dimension 3 and suppose that the scalar curvature $S$ of $g$ is constant. Then, 
(i) there exist two eta-Killing spinors $\psi_{0}, \psi_{1}$ with Killing pair $\left(-\frac{1}{2}, \frac{3}{4}-\frac{S}{8}\right)$ such that $\psi_{\alpha}$ is a section in the bundle $\Sigma_{\alpha}(\alpha=0,1)$.

(ii) If $S \geq-2$, there exists an eta-Killing spinor $\psi \in \Gamma\left(\Sigma=\Sigma_{0} \oplus \Sigma_{1}\right)$ with Killing pair $\left(\frac{-2 \pm \sqrt{4+2 S}}{4}, \frac{4 \mp \sqrt{4+2 S}}{4}\right)$.

We are now ready to apply Theorems 2.1 and 2.2 to Sasakian spin manifolds.

Proposition 3.5. Let $\left(M^{2 m+1}, \phi, \xi, \eta, g\right), m \geq 1$, be a closed Sasakian spin manifold. Let $\beta^{-1}$ be a nondegenerate symmetric tensor field on $M^{2 m+1}$ defined by $\beta^{-1}=\frac{2}{n} I-2 \xi \otimes \eta$. (Note that $\operatorname{div}\left(\beta^{-1}\right)=0$ and $\operatorname{tr}\left(\beta^{-1}\right)=0$.) Let $\lambda_{1} \in \mathbb{R}$ and $\bar{\lambda}_{1} \in \mathbb{R}$ be the first eigenvalue of $D$ and $D_{\beta}$, respectively. Then we have

$$
\lambda_{1}^{2} \geq \frac{n S_{\min }}{4(n-1)}+\frac{n^{2} \bar{\lambda}_{1}^{2}}{4(n-1)^{2}} .
$$

In case that $\lambda_{1} \neq 0$ is nonzero, inequality (3.9) can be rewritten as

$$
\lambda_{1}^{2} \geq \frac{n(n-1) S_{\min }}{4(n-1)^{2}-n^{2} c_{1}^{2}},
$$

where $c_{1}=\bar{\lambda}_{1} / \lambda_{1}$. The limiting case of (3.9) occurs, in case

(i) $n \geq 5$, if and only if there exists an eta-Killing spinor $\psi_{1}$ with Killing pair

$$
\left(\frac{1}{2},-\frac{n}{4}+\frac{S}{4(n-1)}\right), \quad\left(-\frac{1}{2}, \frac{n}{4}-\frac{S}{4(n-1)}\right),
$$

such that $\psi_{1}$ is a first eigenspinor of $D_{\beta}$.

(ii) $n=3$, if and only if there exists an eta-Killing spinor $\varphi_{1}$ with Killing pair

such that $\varphi_{1}$ is a first eigenspinor of $D_{\beta}$.

$$
\left(\frac{-2+\sqrt{4+2 S}}{4}, \frac{4-\sqrt{4+2 S}}{4}\right)
$$

Proof. By Theorem 2.1, the limiting case of (3.9) occurs if and only if there exists an eta-Killing spinor with Killing pair

$$
\left(a_{1}, b_{1}\right)=\left(-\frac{2(n-1) \lambda_{1}+n \bar{\lambda}_{1}}{2 n(n-1)}, \frac{n \bar{\lambda}_{1}}{2(n-1)}\right)
$$

such that it is a first eigenspinor of both $D$ and $D_{\beta}$. Due to Propositions 3.1 and 3.3 we are able to express $a_{1}=-\frac{2(n-1) \lambda_{1}+n \bar{\lambda}_{1}}{2 n(n-1)}$ and $b_{1}=\frac{n \bar{\lambda}_{1}}{2(n-1)}$ in terms of the scalar curvature. Assume that $\left(M^{2 m+1}, \phi, \xi, \eta, g\right)$ is nonEinstein, i.e., $S \neq n(n-1)$. We have to find out which type of eta-Killing spinors, among the various ones in Proposition 3.1 (resp. Proposition 3.3), is a candidate for a first eigenspinor of $D$. Since the case $m \geq 2$ is clear, we consider the 3-dimensional case. Because of the inequality

$$
\left(\frac{3}{4}+\frac{S}{8}\right)^{2}>\left(\frac{1-\sqrt{4+2 S}}{2}\right)^{2}
$$


one finds easily that the eta-Killing spinor with Killing pair

$$
\left(a_{1}, b_{1}\right)=\left(\frac{-2+\sqrt{4+2 S}}{4}, \frac{4-\sqrt{4+2 S}}{4}\right)
$$

is a candidate for a first eigenspinor of $D$, satisfying (3.11) with

$$
\lambda_{1}=\frac{1-\sqrt{4+2 S}}{2}, \quad \bar{\lambda}_{1}=\frac{4-\sqrt{4+2 S}}{3} .
$$

Consequently, the discussion in Remark 2.4 completes the proof.

Remark 3.2. Let $\left(M^{2 m+1}, \phi, \xi, \eta, g\right), m \geq 1$, be a closed Sasakian spin manifold. From inequality (3.9) we see that $\lambda_{1} \neq 0$ is necessarily nonzero if the scalar curvature is positive $S>0$. The statement for the limiting case then gives rise to a question:

Is every eta-Killing spinor with Killing pair (1.8) or (1.9) a first eigenspinor of the Dirac operator when the scalar curvature is positive?

If the scalar curvature $S \leq 0$ is nonpositive, then the first eigenvalue $\lambda_{1}$ is allowed to be zero and the answer to the question is negative. Note that, if $\psi$ is an eta-Killing spinor with Killing pair $\left( \pm \frac{1}{2}, \mp \frac{n}{4} \pm \frac{S}{4(n-1)}\right)$, then its associated eigenvalue $\lambda$ is given by

$$
\lambda=\mp \frac{n(n-1)+S}{4(n-1)} .
$$

There exist 5-dimensional eta-Einstein Sasakian spin manifolds with scalar curvature $S=-4$ that admit harmonic spinors and so the eigenvalue in (3.12) cannot be the first eigenvalue. Examples are certain $S^{1}$-bundles over a 4 dimensional flat torus or quotients of the 5-dimensional Heisenberg group [1, $7,8]$.

To consider an application of Theorem 2.2 to Sasakian spin manifolds, we choose a divergencefree tensor $\gamma^{-1}=I-2 \xi \otimes \eta$.

Proposition 3.6. Let $\left(M^{2 m+1}, \phi, \xi, \eta, g\right), m \geq 1$, be a closed Sasakian spin manifold with positive scalar curvature $S>0$. Let $\gamma^{-1}$ be a nondegenerate symmetric tensor field on $M^{2 m+1}$ defined by $\gamma^{-1}=I-2 \xi \otimes \eta$. Let $\lambda_{1} \neq 0 \in \mathbb{R}$ and $\bar{\lambda}_{1} \in \mathbb{R}$ be the first eigenvalue of $D$ and $D_{\gamma}$, respectively, and denote $c_{1}=\bar{\lambda}_{1} / \lambda_{1}$. Then, for any positive constant $c$ with

$$
\frac{n-2}{n}<c<\frac{n}{n-2},
$$

we have

$$
\lambda_{1}^{2} \geq \frac{c(n-1) S_{\min }}{(n-2) c^{2}+\left(3 n-4-n c_{1}^{2}\right) c+(n-2) c_{1}^{2}} .
$$

The limiting case of (3.14) occurs, in case 
(i) $n \geq 5$, if and only if $c=c_{1}$ and there exists an eta-Killing spinor $\psi_{1}$ with Killing pair

$$
\left(\frac{1}{2},-\frac{n}{4}+\frac{S}{4(n-1)}\right), \quad\left(-\frac{1}{2}, \frac{n}{4}-\frac{S}{4(n-1)}\right),
$$

such that $\psi_{1}$ is a first eigenspinor of $D_{\gamma}$.

(ii) $n=3$, if and only if $c=c_{1}$ and there exists an eta-Killing spinor $\varphi_{1}$ with Killing pair

$$
\left(\frac{-2+\sqrt{4+2 S}}{4}, \frac{4-\sqrt{4+2 S}}{4}\right)
$$

such that $\varphi_{1}$ is a first eigenspinor of $D_{\gamma}$.

Proof. By Theorem 2.2, the limiting case of (3.14) occurs if and only if $c=c_{1}$ and there exists an eta-Killing spinor with Killing pair

$$
\left(a_{1}, b_{1}\right)=\left(-\frac{\left(c_{1}+1\right) \lambda_{1}}{2(n-1)}, \frac{\left(n c_{1}-n+2\right) \lambda_{1}}{2(n-1)}\right)
$$

such that it is a first eigenspinor of both $D$ and $D_{\gamma}$. Proceeding as in the proof of Proposition 3.5, one establishes the proposition.

Remark 3.3. Inequality (3.14) is not generally sharper than Friedrich's inequality (1.5), i.e.,

$$
\frac{c(n-1) S_{\min }}{(n-2) c^{2}+\left(3 n-4-n c_{1}^{2}\right) c+(n-2) c_{1}^{2}}>\frac{n S_{\min }}{4(n-1)}
$$

holds if and only if

$$
(n c-n+2)\left[(n-2) c-n c_{1}^{2}\right]<0
$$

is satisfied.

Acknowledgements. The author thanks Thomas Friedrich for pointing out that the question in Remark 3.2 has no meaning for Sasakian spin manifolds with negative scalar curvature.

\section{References}

[1] I. Agricola and Th. Friedrich, The Casimir operator of a metric connection with skewsymmetric torsion, J. Geom. Phys. 50 (2004), no. 1-4, 188-204.

[2] C. Bär, Real Killing spinors and holonomy, Comm. Math. Phys. 154 (1993), no. 3, 509-521.

[3] H. Baum, Th. Friedrich, R. Grunewald, and I. Kath, Twistors and Killing Spinors on Riemannian Manifolds, Teubner, Leipzig/Stuttgart, 1991.

[4] C. P. Boyer, K. Galicki, and P. Matzeu, On eta-Einstein Sasakian geometry, Comm. Math. Phys. 262 (2006), no. 1, 177-208.

[5] Th. Friedrich, Der erste Eigenwert des Dirac-Operators einer kompakten, Riemannschen Mannigfaltigkeit nichtnegativer Skalarkrümmung, Math. Nachr. 97 (1980), 117146.

[6] _ Dirac Operators in Riemannian Geometry, Graduate Studies in Mathematics, vol. 25, American Mathematical Society, Providence, RI, 2000. 
[7] Th. Friedrich and S. Ivanov, Parallel spinors and connections with skew-symmetric torsion in string theory, Asian J. Math. 6 (2002), no. 2, 303-335.

[8] _ Almost contact manifolds, connections with torsion, and parallel spinors, J. Reine Angew. Math. 559 (2003), 217-236.

[9] Th. Friedrich and E. C. Kim, The Einstein-Dirac equation on Riemannian spin manifolds, J. Geom. Phys. 33 (2000), no. 1-2, 128-172.

[10] Eigenvalues estimates for the Dirac operator in terms of Codazzi tensors, Bull. Korean Math. Soc. 45 (2008), no. 2, 365-373.

[11] K.-D. Kirchberg, An estimation for the first eigenvalue of the Dirac operator on closed Kahler manifolds of positive scalar curvature, Ann. Global Anal. Geom. 4 (1986), no. 3, 291-325.

[12] M, Wang, Parallel spinors and parallel forms, Ann. Global Anal. Geom. 7 (1989), no. $1,59-68$.

Department of Mathematics

College of Education

Andong National University

ANDONG 760-749, KoREA

E-mail address: eckim@andong.ac.kr 